\title{
Establishment of a serological molecular model for the early diagnosis and progression monitoring of bone metastasis in lung cancer
}

Xiaoyan Teng ${ }^{1}$, Lirong $\mathrm{Wei}^{1}$, Liming Han ${ }^{1}$, Daliu Min ${ }^{2^{*}}$ and Yuzhen Du ${ }^{1^{*}}$

\begin{abstract}
Background: The prognosis is very poor for lung cancer patients with bone metastasis. Unfortunately, a suitable method has yet to become available for the early diagnosis of bone metastasis in lung cancer patients. The present work describes an attempt to develop a novel model for the early identification of lung cancer patients with bone metastasis risk.

Methods: As the test group, 205 primary lung cancer patients were recruited, of which 127 patients had bone metastasis; the other 78 patients without bone metastasis were set as the negative control. Additionally, 106 healthy volunteers were enrolled as the normal control. Serum levels of several cytokines in the bone microenvironment (CaN, OPG, PTHrP, and IL-6) and bone turnover markers (tP1NP, $\beta-C T x$ ) were detected in all samples by ECLIA or ELISA assay. Receiver operating characteristic (ROC) curves and multivariate analyses were performed to evaluate diagnostic abilities and to assess the attributable risk of bone metastasis for each of these indicators; the diagnostic model was established via logistic regression analysis. The prospective validation group consisted of 44 patients with stage IV primary lung cancer on whom a follow-up of at least 2 years was conducted, during which serum bone biochemical marker concentrations were monitored.
\end{abstract}

Results: The serological molecular model for the diagnosis of bone metastasis was logit (p). ROC analysis showed that when logit $(p)>0.452$, the area under curve of the model was 0.939 (sensitivity: 85.8\%, specificity: 89.7\%). Model validation demonstrated accuracy with a high degree of consistency (specificity: $85.7 \%$, specificity: $87.5 \%$, Kappa: 0.770). The average predictive time for bone metastasis occurrence of the model was 9.46 months earlier than that of the bone scan diagnosis. Serum OPG, PTHrP, tP1NP, $\beta-C T x$, and the diagnostic model logit (p) were all positively correlated with bone metastasis progression $(P<0.05)$.

Conclusions: This diagnostic model has the potential to be a simple, non-invasive, and sensitive tool for diagnosing the occurrence and monitoring the progression of bone metastasis in patients with lung cancer.

Keywords: Bone microenvironment cytokines, Bone turnover markers, Diagnostic risk factors, Bone metastasis, Lung cancer

\footnotetext{
*Correspondence: Mindaliu1969@163.com; sd_duyz@Sumhs.edu.cn;

duyuzhen2005@163.com

2 Department of Oncology, Shanghai Jiao Tong University Affiliated Sixth

People's Hospital of Shanghai, Shanghai 200233, China

${ }^{1}$ Department of Laboratory Medicine, Shanghai Jiao Tong University

Affiliated Sixth People's Hospital of Shanghai, Shanghai 200233, P.R. China
}

C The Author(s). 2020 Open Access This article is licensed under a Creative Commons Attribution 4.0 International License, which permits use, sharing, adaptation, distribution and reproduction in any medium or format, as long as you give appropriate credit to the original author(s) and the source, provide a link to the Creative Commons licence, and indicate if changes were made. The images or other third party material in this article are included in the article's Creative Commons licence, unless indicated otherwise in a credit line to the material. If material is not included in the article's Creative Commons licence and your intended use is not permitted by statutory regulation or exceeds the permitted use, you will need to obtain permission directly from the copyright holder. To view a copy of this licence, visit http://creativecommons.org/licenses/by/4.0/ The Creative Commons Public Domain Dedication waiver (http://creativecommons.org/publicdomain/zero/1.0/) applies to the data made available in this article, unless otherwise stated in a credit line to the data. 


\section{Background}

Lung cancer patients are prone to developing bone metastasis accompanied by bone-related events such as bone pain, fracture, hypercalcemia, and nerve compression, which seriously affect patient quality of life $[1,2]$. Clinically, the diagnosis of bone metastasis from lung cancer relies mainly on clinical symptoms and bone scans. There is no effective way to control bone destruction when tumour metastasis results in radiographically detectable lesions [3]. Therefore, the establishment of a non-invasive method for the early diagnosis of bone metastasis could significantly improve the prognosis and quality of life of patients with lung cancer. When bone metastasis occurs, osteoblasts in the bone microenvironment (BME) secrete calcineurin ( $\mathrm{CaN})$ and osteoprotegerin (OPG) $[4,5]$, and tumour cells secrete parathyroid hormone-related peptide (PTHrP) and interleukin- 6 (IL-6) [6, 7]. These cytokines interact with tumour cells, osteoblasts, osteoclasts, and stromal cells in the BME to promote osteogenic differentiation and bone resorption. When the delicate balance of bone metabolism is broken, bone turnover markers in the blood, including the bone formation marker tP1NP and the bone resorption marker $\beta$-CTx, are significantly increased $[8,9]$. BME cytokines (CaN, OPG, PTHrP, and IL-6) and bone turnover markers ( $\mathrm{tP} 1 \mathrm{NP}$ and $\beta-\mathrm{CTx}$ ) can be detected in the serum of patients $[10,11]$. These serum indicators can serve as special serum bone biochemical markers for bone metastasis. Therefore, changes in the concentrations of these bone biochemical markers may be associated with the progression of tumour bone metastasis.

In this study, the serum concentrations of BME cytokines (CaN, OPG, PTHrP, and IL-6) and bone turnover markers (tP1NP and $\beta$-CTx) were analysed in 205 lung cancer patients, and these bone biochemical markers were comprehensively analysed in relation to bone metastasis in lung cancer. The diagnostic values were assessed and a serological molecular model was established to provide an experimental basis for the early diagnosis and progression monitoring of lung cancer bone metastasis.

\section{Methods}

\section{Patients' clinical characteristics}

This study was approved by the Ethics Committee of the East Campus of Shanghai Sixth People's Hospital, affiliated with Shanghai Jiaotong University. All participants in the study provided written informed consent prior to recruitment.

Patients with stage IV primary lung cancer that were admitted to the East Campus of Shanghai Sixth People's Hospital from October 2015 to August 2016 were enrolled. After excluding patients who had received chemotherapy, radiotherapy, immunotherapy, bisphosphonate therapy, or other treatment within 3 months prior enrolment and those with endocrine disorders or immune or metabolic diseases, a total of 205 patients were enrolled in the study. Follow-up was conducted until October 2018, with a minimum follow-up period of 2 years. Of the enrolled patients, 127 (bone metastasis group) had bone metastasis, and the other 78 (non-bone metastasis group) did not have bone metastasis, as determined on the basis of pathology or bone scans. Bone scan examination was performed every 6 months to monitor the progression of bone metastasis in all patients. In addition, 106 healthy volunteers were recruited as normal controls. The control group was age- and gender-matched with the test group. The clinical characteristics of the patients and healthy volunteers are shown in Supplementary Table 1.

The prospective validation study included 44 patients with stage IV primary lung cancer who underwent a complete surgical resection between October 2015 and August 2016 and were followed until October 2018, for a minimum of 2 years. All patients had been diagnosed with non-bone metastasis by pathology or bone scan at the initial presentation. Bone scan examination was performed every 6 months to monitor bone metastasis in all patients. The clinical characteristics of 44 patients, including age, gender, histological type, and stage are shown in Supplementary Table 2. Changes in the serum levels of bone biochemical markers were monitored during follow-up. Over the follow-up period, bone metastasis occurred in some patients, and serum samples obtained at the time of bone metastasis diagnosis were used as endpoint follow-up samples. For the patients without bone metastasis, the serum obtained after 2 years of follow-up was used as the endpoint follow-up sample.

\section{Collection and determination of the serum samples}

A total of $2-4 \mathrm{ml}$ of fasting venous blood from patients and healthy volunteers was collected in the morning and left at $25^{\circ} \mathrm{C}$ for $30 \mathrm{~min}$. The supernatant serum was obtained by centrifugation at $4000 \mathrm{~g}$ for $10 \mathrm{~min}$, and then the serum samples from the individuals were transferred to new tubes in $500 \mu \mathrm{l}$ aliquots and stored at $-80^{\circ} \mathrm{C}$.

The serum concentrations of tP1NP (03141071190), $\beta$ CTx (11972308122), and IL-6 (05109442190) were quantified using a Diagnostics Cobas E601 fully automated electrochemiluminescence immunoassay (ECLIA) (Swiss Roche) and its original reagents. The inter-assay precision CVs of tP1NP, $\beta$-CTx, and IL- 6 were less than 3.2, 4.7 , and $3.9 \%$, respectively. The intra-assay precision CVs of tP1NP, $\beta$-CTx and IL-6 were less than 8.7, 5.7, and $5.1 \%$, respectively.

The serum concentrations of CaN (BYS10821B), OPG (BYS10849B), and PTHRP (BYS10753B) were measured quantitatively by an enzyme-linked immunosorbent 
assay (ELISA) kit (Shanghai Boyan Biotech, China). All of the methods were performed in accordance with the manufacturer's instructions. The optical densities (OD) at $450 \mathrm{~nm}$ and $622 \mathrm{~nm}$ were detected with a microplate reader (Biotech, Winooski, VT, USA), and the repeatability CV was less than $15 \%$.

\section{Statistical analysis}

MedCalc software (Medcalc, Mariakerke, Belgium) and GraphPad Prism (San Diego, CA, USA) were used for statistical analysis and mapping. The serum levels of bone biochemical markers of the two groups were compared with the nonparametric Kruskal Wallis test. Spearman correlation analysis was used to assess the correlation between the serum concentrations of these indicators. An ROC curve analysis was used to evaluate the diagnostic value of each of these indicators for bone metastasis in lung cancer. The optimal Youden index method was used to determine the cut-off value of these indicators for the diagnosis of bone metastasis. In addition, univariate and multivariate logistic regression analyses were performed to assess the risk attributed by each of these markers for the development of bone metastasis in lung cancer and to establish a serological molecular model for the diagnosis of bone metastasis in lung cancer. The Hosmer-Lemeshow test and an ROC curve analysis were used to evaluate the model-fitting effect and diagnostic accuracy, and the Kappa test was used to evaluate the consistency of the follow-up bone scan diagnosis results with the model diagnosis results. In the follow-up study, the change in serum index was calculated with the following equation: $[\%$ year change (median $)]=[($ followup level - initial level) / (year * initial level)]. The nonparametric Mann-Whitney test was used to compare the initial levels, follow-up levels, \% year change, and logit (p) between the bone metastasis group and the non-bone metastasis group. The nonparametric Wilcoxon test was used to compare differences within the bone metastasis group and the non-bone metastasis group, including initial levels, follow-up levels, and model logit (p). A value of $P<$ 0.05 was considered statistically significant.

\section{Clinical practice points}

1. Patients with lung cancer have high serum levels of bone biochemical markers (BME cytokines and bone turnover markers) and are prone to bone metastasis; there is no existing method that can diagnose bone metastasis early or monitor its occurrence in lung cancer patients.

2. For patients with stage IV lung cancer, those with high serum concentrations of BME cytokines and bone turnover markers were significantly associated with the occurrence of bone metastasis.
3. In addition to clinical pathological factors, this serum bone biochemical marker model could be a simple, non-invasive, and sensitive tool for the diagnosis and progression monitoring of bone metastasis in patients with lung cancer.

\section{Results}

Serum levels of BME cytokines and bone turnover markers in lung cancer patients with or without bone metastasis disease

Compared with the healthy control, the serum concentrations of BME cytokines and bone turnover markers were significantly higher in the lung cancer bone metastasis group $(P<0.05)$. In non-bone the metastasis group, serum levels of all serum bone biochemical markers were slightly higher than those of the healthy controls $(P<0.05)$ (Fig. 1 A/B/D), except for PTHrP $(P>0.05)$ (Fig. 1 C).

Compared with those in the non-bone metastasis group, the serum levels of all BME cytokines in the bone metastasis group were significantly elevated, including $\mathrm{CaN}, \mathrm{OPG}$, PTHrP, and IL-6 $(P<0.05)$ (Fig. 1 A/B/C/D). Similar results were observed for the bone turnover markers. The serum levels of tP1NP and $\beta$-CTx in the bone metastasis group were significantly higher than those in the nonbone metastasis group $(P<0.05)($ Fig. $2 \mathrm{~A} / \mathrm{B})$.

\section{Diagnostic values of serum BME cytokines and bone turnover markers for bone metastasis in patients with lung cancer}

The ROC curve analysis showed that the serum BME cytokines $\mathrm{CaN}$, OPG, and PTHrP had high diagnostic values for bone metastasis in lung cancer. The areas under curve (AUC) for these indicators were $0.808,0.825$, and 0.854 , and the cut-off values were $1257.80 \mathrm{ng} / \mathrm{ml}, 1547.00$ $\mathrm{ng} / \mathrm{ml}$, and $322.30 \mathrm{pg} / \mathrm{ml}$, respectively. The diagnostic ability of serum IL-6 was limited; the AUC was 0.652 , and the cutoff value was $14.36 \mathrm{pg} / \mathrm{ml}$. The AUC for the four cytokines combined (CaN, OPG, PTHrP, and IL-6) was $0.898[P<$ $0.001,95 \%$ CI $(0.848-0.936)]$, with a sensitivity of $79.49 \%$ and a specificity of $89.76 \%$ (Fig. 1E/F, Tables 1 and 2). The diagnostic ability of the cytokine combination was superior to that of each cytokine alone.

The serum bone turnover markers tP1NP and $\beta$-CTx also had high diagnostic values for bone metastasis in lung cancer. The AUCs of tP1NP and $\beta$-CTx were 0.838 and 0.812 , and the cut-off values were $93.59 \mu \mathrm{g} / \mathrm{L}$ and $619.70 \mathrm{ng} / \mathrm{ml}$, respectively. The AUC of tP1NP combined with $\beta$-CTx was $0.851[P<0.001,95 \%$ CI $(0.794-0.897)]$, the sensitivity was $71.79 \%$, and the specificity was 78.74\% (Fig. 2 C/D, Tables 1 and 2).

The combination of bone turnover markers showed stronger diagnostic performance than each one used alone, but the sensitivity was limited (Tables 1 and 2). 

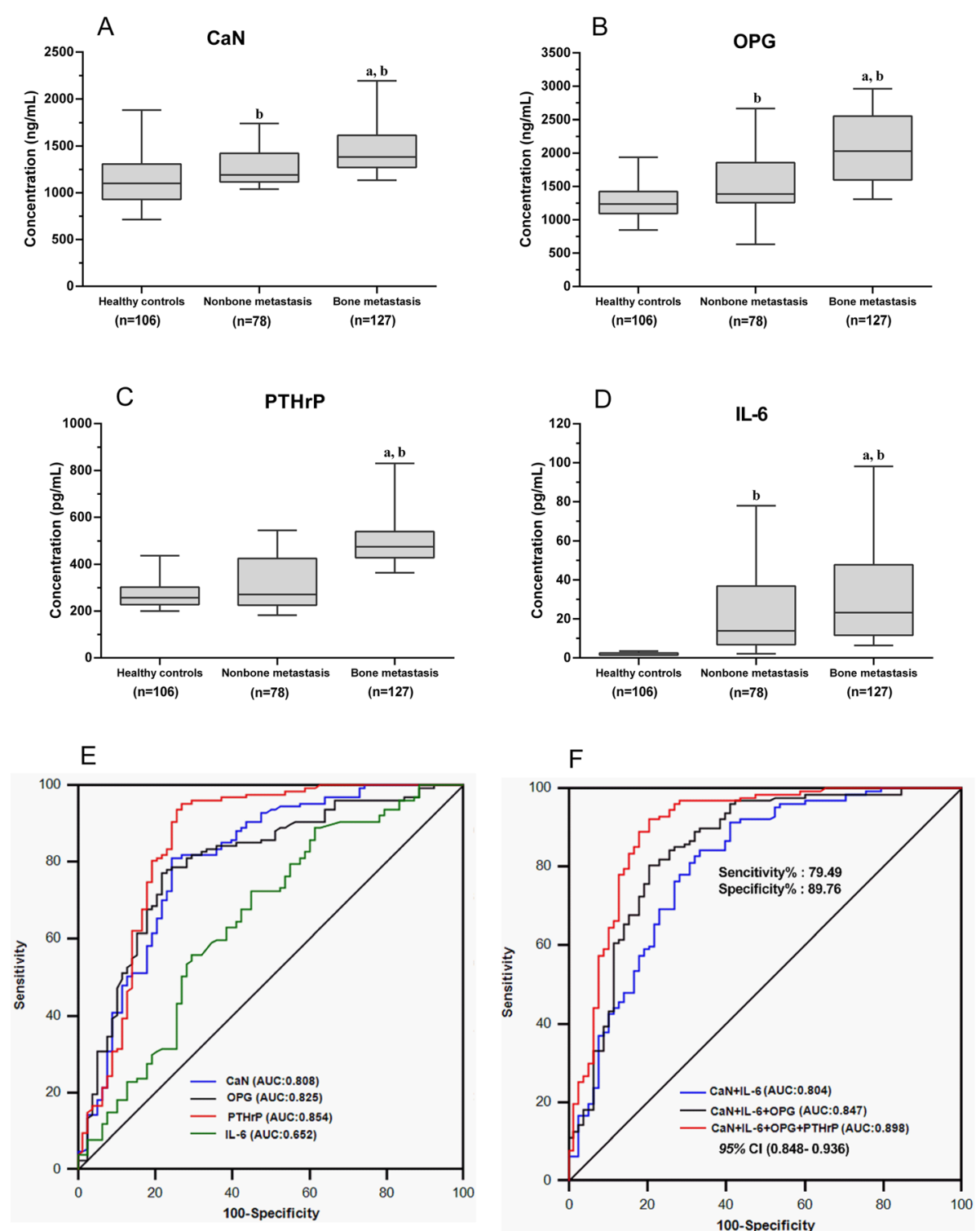

Fig. 1 Serum levels of BME cytokines and their diagnostic abilities for bone metastasis in stage IV lung cancer patients. $\mathbf{a}, \mathbf{b}, \mathbf{c}, \mathbf{d}$ Box plots of the serum levels of the BME cytokines CaN, OPG, PTHrP, and IL-6 in three groups, including the bone metastasis group $(n=127)$, the non-bone metastasis group ( $n=$ 78), and the healthy control group $(n=106)$. From the bottom up, the boxes indicate the 25th, 50th (median), and 75th percentiles, while the bars indicate the 10th and 90th percentiles, respectively. Significance levels were obtained from the nonparametric Kolmogorov-Smirnov test: (a) $P<0.05$ : vs non-bone metastasis, (b) $P<0.05$ : vs healthy controls. e ROC analysis of the diagnostic abilities of serum bone microenvironment cytokines for bone metastasis. $\mathbf{f}$ ROC analysis of the diagnostic efficiencies of different combinations of the bone microenvironment cytokines for bone metastasis

\section{Correlation between serum levels of BME cytokines and bone turnover markers}

Serum BME cytokines and bone turnover markers were analysed in 205 patients with stage IV primary lung cancer. A positive correlation was found between the four cytokines ( $\mathrm{r}$ values ranged from 0.289 to $0.674, P<$ 0.001) (Supplementary Table 3 ), especially between $\mathrm{CaN}$ and PTHrP $(\mathrm{r}=0.674, P<0.001)$ (Fig. 3A). Interestingly, there was a positive correlation between PTHrP and tP1NP $(\mathrm{r}=0.428, P<0.001)$ (Fig. 3B).
Establishment and validation of a serological molecular model for the diagnosis of bone metastasis in lung cancer

Inclusive indicators included the serum levels of BME cytokines and bone turnover markers, age, sex, pathological type, stage, and bone scan results of the patients (see Supplementary Table 4 for details). The patients were grouped according to cut-off values and subjected to univariate and multivariate regression analyses. Serum CaN, OPG, PTHrP, IL-6, tP1NP, $\beta$-CTx, and tissue type 

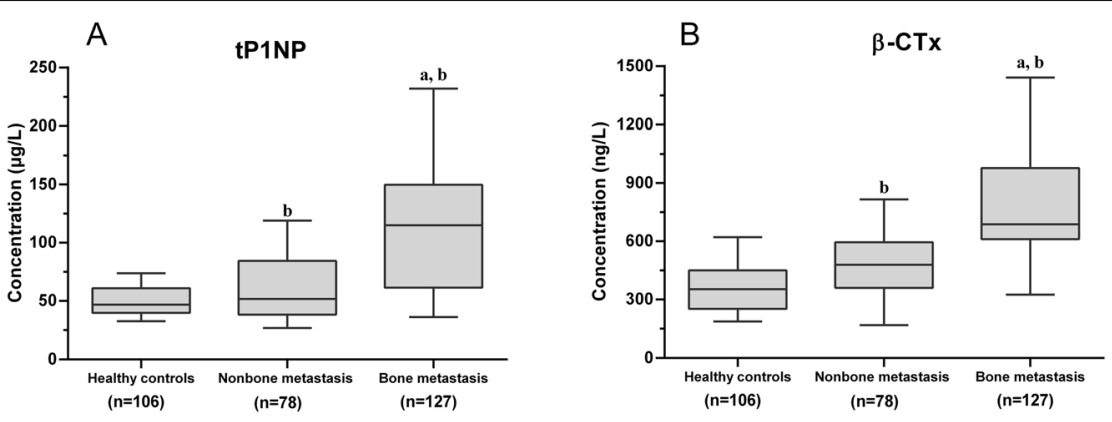

C
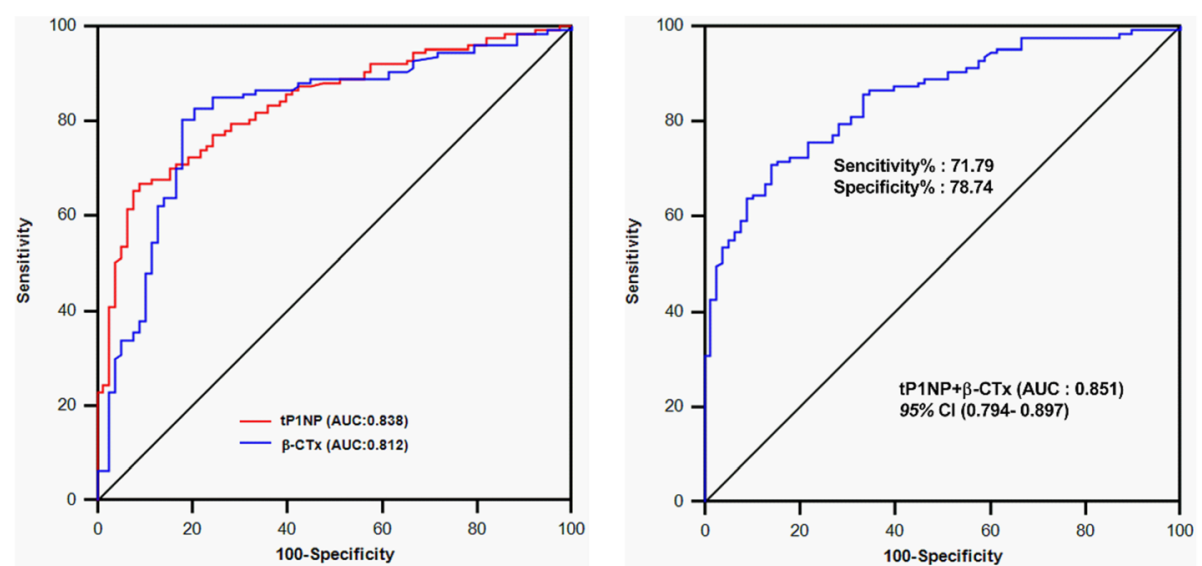

Fig. 2 Serum levels of bone turnover markers and their abilities to diagnose bone metastasis in stage IV lung cancer patients. a, b Box plot distribution of the serum levels of the bone turnover markers tPINP and $\beta$-CTx from three groups, including the bone metastasis group ( $n=127$ ), the non-bone metastasis group $(n=78)$, and the healthy control group $(n=106)$. For details of the box plots, see the legend of Fig. 1. c ROC analysis of the ability of the serum bone turnover markers IP1NP and $\beta-C T x$ to diagnose bone metastasis. $\mathbf{d}$ ROC analysis of the diagnostic efficiency of the combination of tP1NP and $\beta-C T x$

were the univariate diagnostic factors for bone metastasis in lung cancer $(P<0.05)$; age and sex were the only two excluded factors. All variables with $P<0.05$ in the univariate analysis were analysed by multivariate logistic regression to determine whether these serum markers were independently related to the diagnosis of bone metastasis in lung cancer. Multivariate logistic regression analysis indicated that only OPG $(P<0.001)$, PTHrP $(P<0.001)$, tP1NP $(P=0.001)$, and $\beta$-CTx $(P<0.001)$ were independent diagnostic factors for bone metastasis in lung cancer, while $\mathrm{CaN}$ and IL-6 were not (Fig. 4A and Supplementary Table 4).

Table 1 Diagnostic ability of serum bone biomarkers to distinguish lung cancer patients with bone metastasis from those without bone involvement

\begin{tabular}{|c|c|c|c|c|c|c|c|}
\hline Marker & AUC & $95 \% \mathrm{Cl}$ & $\begin{array}{l}\text { Cut-off } \\
\text { level }\end{array}$ & $\begin{array}{l}\text { Sensitivity } \\
\%\end{array}$ & $\begin{array}{l}\text { Specificity } \\
\%\end{array}$ & $P$ value & $\begin{array}{l}\text { Diagnostic } \\
\text { accuracy (\%) }\end{array}$ \\
\hline \multicolumn{8}{|l|}{ BME cytokine } \\
\hline $\mathrm{CaN}(\mathrm{ng} / \mathrm{mL})$ & 0.81 & $0.747-0.859$ & 1257.80 & 81.10 & 75.60 & $<0.001$ & 78.74 \\
\hline OPG (ng/mL) & 0.83 & $0.760-0.856$ & 1547.00 & 77.20 & 78.20 & $<0.001$ & 77.30 \\
\hline PTHrP (pg/mL) & 0.85 & $0.798-0.899$ & 322.30 & 95.30 & 73.10 & $<0.001$ & 51.70 \\
\hline IL-6 (pg/mL) & 0.65 & $0.583-0.717$ & 14.36 & 72.40 & 55.10 & $<0.001$ & 65.90 \\
\hline \multicolumn{8}{|c|}{ Bone turnover marker } \\
\hline tP1NP $(\mu \mathrm{g} / \mathrm{L})$ & 0.84 & $0.780-0.885$ & 93.59 & 66.90 & 91.00 & $<0.001$ & 74.71 \\
\hline$\beta-C T \times(n g / L)$ & 0.81 & $0.749-0.865$ & 619.70 & 80.30 & 82.10 & $<0.001$ & 83.91 \\
\hline
\end{tabular}


Table 2 Diagnostic efficiency of different combinations of serum bone biomarkers to distinguish lung cancer patients with bone metastasis from those without bone involvement

\begin{tabular}{|c|c|c|c|c|c|c|}
\hline & & & Sensitivity & Specificity & & Diagnostic \\
\hline Combination & AUC & $95 \% \mathrm{Cl}$ & $\%$ & $\%$ & $P$ value & accuracy (\%) \\
\hline \multicolumn{7}{|l|}{ BME cytokines } \\
\hline $\mathrm{CaN}+\mathrm{IL}-6$ & 0.804 & $0.743-0.856$ & 60.26 & 84.25 & $<0.001$ & 75.12 \\
\hline $\mathrm{CaN}+\mathrm{IL}-6+\mathrm{OPG}$ & 0.847 & $0.790-0.893$ & 74.36 & 84.25 & $<0.001$ & 80.49 \\
\hline $\mathrm{CaN}+\mathrm{IL}-6+\mathrm{OPG}+\mathrm{PTHrP}$ & 0.898 & $0.848-0.936$ & 79.49 & 89.76 & $<0.001$ & 85.63 \\
\hline \multicolumn{7}{|l|}{ Bone turnover markers } \\
\hline $\operatorname{tP} 1 \mathrm{NP}+\beta-C T x$ & 0.851 & $0.794-0.897$ & 71.79 & 78.74 & $<0.001$ & 76.1 \\
\hline \multicolumn{7}{|c|}{ Independent diagnostic risk factors } \\
\hline $\mathrm{OPG}+\mathrm{PTHrP}$ & 0.893 & $0.842-0.931$ & 78.21 & 90.55 & $<0.001$ & 76.1 \\
\hline $\mathrm{OPG}+\mathrm{PTHrP}+\mathrm{tP} 1 \mathrm{NP}$ & 0.924 & $0.864-0.952$ & 81.69 & 84.47 & $<0.001$ & 83.91 \\
\hline $\mathrm{OPG}+\mathrm{PTH}+\mathrm{P}+\mathrm{tP} 1 \mathrm{NP}+\beta-\mathrm{CT} x$ & 0.94 & $0.898-0.968$ & 84.62 & 88.98 & $<0.001$ & 87.32 \\
\hline
\end{tabular}

ROC analysis showed that the combination of four independent diagnostic factors, OPG, PTHrP, tP1NP, and $\beta$-CTx, had the highest diagnostic efficiency, with a diagnostic accuracy of $87.32 \%$. The AUC of this combination was $0.940[P<0.001,95 \% \mathrm{CI}(0.898-0.968)]$ (Fig. 4B and Table 2). Multivariate logistic regression analysis established a molecular model for bone metastasis diagnosis: $\operatorname{logit}(\mathrm{p})=-8.4988+0.0211 * \operatorname{tP} 1 \mathrm{NP}+0.0079 * \mathrm{PTHrP}$ $+0.0016^{*}$ OPG $+0.0021 * \beta-C T x$. The Hosmer-Lemeshow test showed that $P=0.825$, which indicated a good model fit. When logit $(\mathrm{p})>0.452$, the sensitivity and specificity of the predictive model were 85.8 and $89.7 \%$, respectively (Fig. 5A).

To verify the accuracy and effectiveness of the predictive model for bone metastasis, 44 patients with stage IV primary lung cancer without bone metastasis were predicted by the model and followed up for at least 2 years. During the follow-up period, serum levels of OPG, PTHrP, tP1NP, and $\beta$-CTx were continuously monitored, along with the results of bone scans. Of the 44

Table 3 The initial levels of serum bone bimarkers in lung cancer patients who developed bone metastasis and the others who remained free of bone metastasis during follow-up

\begin{tabular}{llll}
\hline & \multicolumn{2}{l}{ Serum bone marker at initial level } & \\
\cline { 2 - 3 } Marker & Yes $(n=16)$ & No $(n=28)$ & P value \\
\hline BME cytokine & & & \\
CaN $(\mathrm{ng} / \mathrm{mL})$ & $1263.54(1713.01)$ & $1287.27(1418.43)$ & 0.625 \\
OPG $(\mathrm{ng} / \mathrm{mL})$ & $1784.69(2319.0)$ & $1638.18(1789.94)$ & 0.105 \\
PTHrP $(\mathrm{pg} / \mathrm{mL})$ & $401.12(476.32)$ & $380.38(431.93)$ & 0.28 \\
IL-6 $(\mathrm{pg} / \mathrm{mL})$ & $23.73(30.01)$ & $23.29(45.19)$ & 0.136 \\
Bone turnover marker & & \\
tP1NP $(\mu \mathrm{g} / \mathrm{L})$ & $80.83(101.60)$ & $71.06(81.62)$ & 0.11 \\
$\beta-C T x(\mathrm{ng} / \mathrm{L})$ & $610.0(894.06)$ & $489.58(624.24)$ & 0.081 \\
\hline
\end{tabular}

Data are expressed as median (interquartile) patients, based on the model, 18 patients had high scores and were predicted to develop bone metastasis, and 26 patients were predicted not to develop the disease. Follow-up results showed that 16 patients developed bone metastasis. The specificity and sensitivity of the predictive model were 85.7 and $87.5 \%$, respectively, and the Kappa value was 0.770 , which showed that the predictive results of the model were highly consistent with the bone scan results (Fig. 5B), but the average predictive time of the serum model was 9.46 months earlier than that of the bone scan (Fig. 6A).

\section{The roles of BME cytokines, bone turnover markers, and diagnostic models in monitoring the progress of bone metastasis}

During the follow-up period, the concentrations of BME cytokines and bone turnover markers in serum samples at the start and end of the follow-up period were measured and recorded as the initial and follow-up levels, respectively.

During the follow-up of 44 patients, 16 patients with lung cancer had bone metastasis (bone metastasis group). The follow-up levels of these serum indicators were significantly higher than their initial levels, except for IL-6 (Table 3). The other 28 patients without bone metastasis (non-bone metastasis group) showed only slight increases in the serum levels of these indicators $(P<0.05)$. The \% year changes of serum OPG $(P=0.001)$, PTHrP $(P=$ $0.011)$, tP1NP $(P=0.002)$, and $\beta$-CTx $(P=0.027)$ in patients with bone metastasis were significantly higher than those in patients without bone metastasis, but there was no significant difference in serum CaN or IL-6 (Fig. 6B). Therefore, the changes in serum concentrations of OPG, PTHrP, $\mathrm{tP} 1 \mathrm{NP}$, and $\beta$-CTx are related to the progression of bone metastasis in lung cancer patients.

In addition, the diagnostic model logit (p) of patients with bone metastasis from lung cancer was significantly 

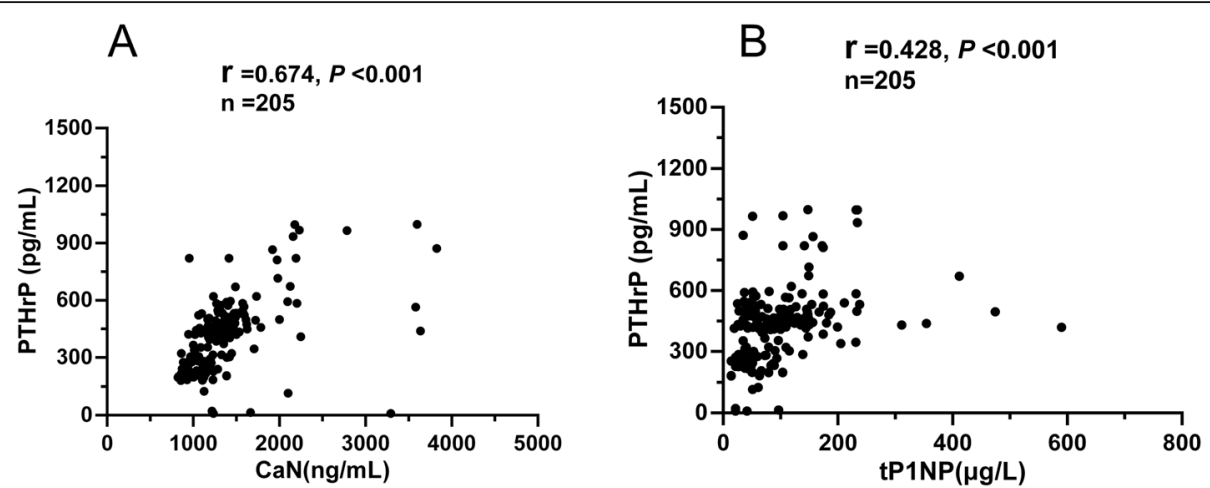

Fig. 3 Correlations between serum levels for BME cytokines and bone turnover markers in 205 patients with stage IV primary lung cancer. a Correlation between serum levels of the bone microenvironment cytokines PTHrP and CaN. b Correlation between serum levels of the bone microenvironment cytokine PTHrP and bone turnover marker tP1NP

higher than that of lung cancer patients without bone metastasis $(P<0.001)$. In addition, the diagnostic logit (p) of bone metastasis in lung cancer patients increased over time $(P<0.001)$ (Fig. 6C).

\section{Discussion}

BME cytokines and bone turnover markers are involved in the early survival mechanisms of tumour cells in bone tissue, such as colonization, dormancy, micrometastasis, and metastasis formation [12]. In this study, serum BME cytokines (CaN, OPG, PTHrP, and IL-6) and bone turnover markers (tP1NP and $\beta$-CTx) were measured in lung cancer patients. The concentrations of these markers in the bone metastasis group were significantly higher than those in the non-bone metastasis group and the healthy control group. An ROC curve analysis showed that serum CaN, OPG, PTHRP, tP1NP, and $\beta$-CTx were valuable in the diagnosis of bone metastasis in lung cancer. Univariate and multivariate logistic regression analyses showed that serum OPG, PTHrP, tP1NP, and $\beta$ $\mathrm{CTx}$ were independent diagnostic factors for bone metastasis in lung cancer. Therefore, a serum molecular model for the diagnosis of bone metastasis in lung cancer was established. Forty-four patients with lung cancer were followed up to verify the diagnostic value of this serum model for bone metastasis in lung cancer. It was found that the model could accurately indicate the occurrence of bone metastasis in lung cancer, and the
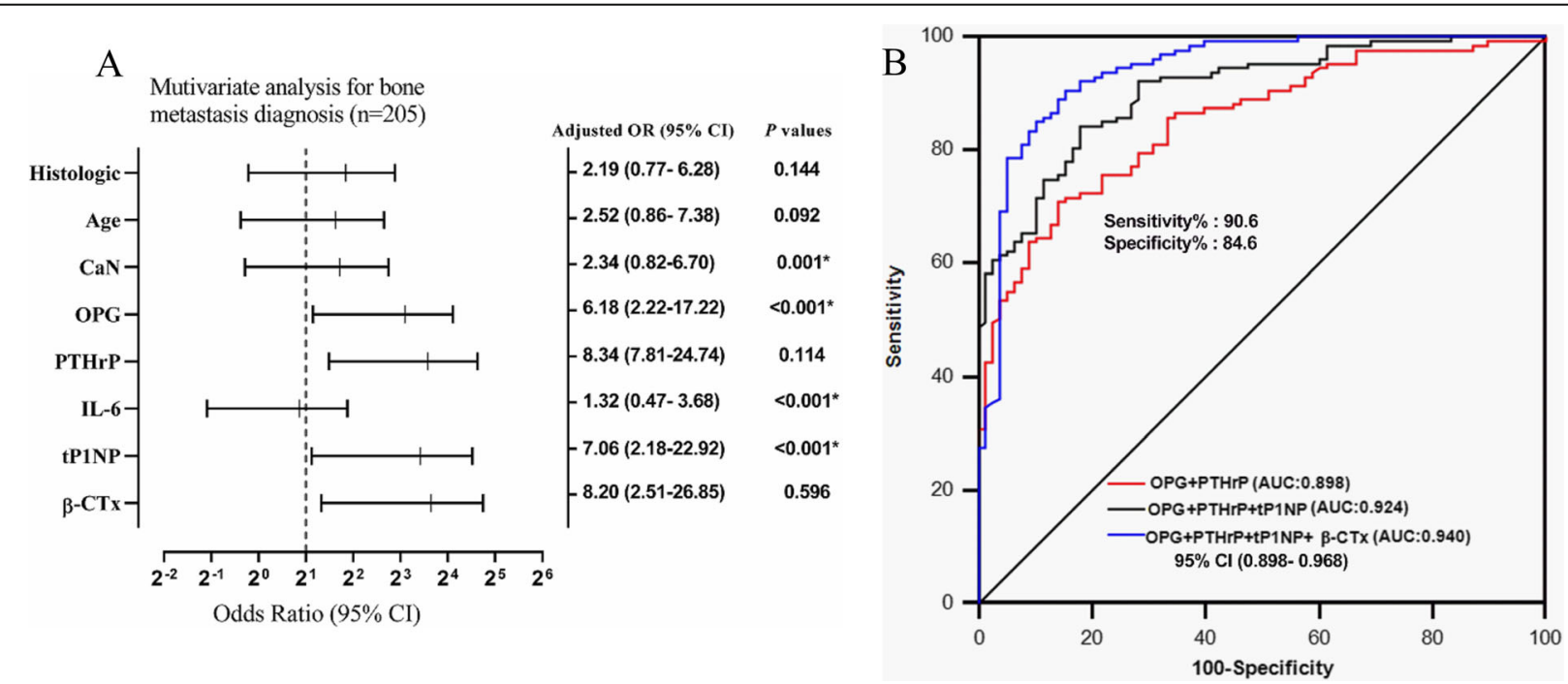

Fig. 4 Evaluation of the attributable risk and diagnostic efficiency of these parameters for bone metastasis in 205 patients with stage IV primary lung cancer. a Multivariate logistic regression analysis to evaluate the attributable risk of these parameters for bone metastasis. OR values were presented by log2 transformation. OR: odds ratio, Cl: confidence interval. ${ }^{*} P<0.05$ was considered statistically significant. b ROC analysis to assess the diagnostic efficiency for bone metastasis of different combinations of the four independent diagnostic risk factors, including BME cytokines (OPG and PTHrP) and bone turnover markers (tP1NP and $\beta-C T x)$ 
A

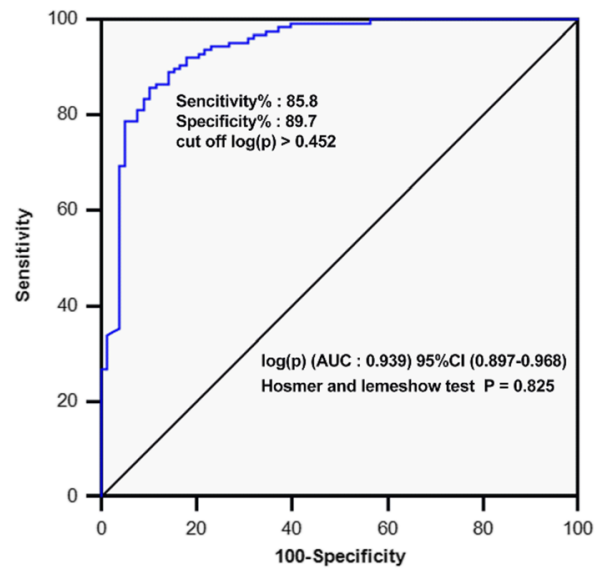

B

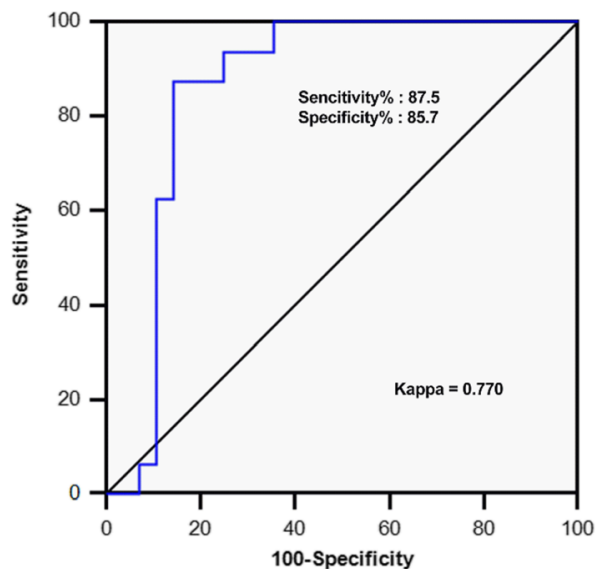

Fig. 5 Diagnostic efficiency of the diagnostic model for bone metastasis in patients with stage IV primary lung cancer. a Evaluation of the model fitting degree and diagnostic accuracy of the model. $\mathbf{b}$ ROC analysis of consistency and effectiveness in the diagnosis of bone metastasis of the model

diagnosis time of this model for bone metastasis was 9.46 (5.00-17.63) months earlier than that of bone scan examination. In addition, the results also suggested that serum OPG, PTHrP, tP1NP, and $\beta$-CTx along with the serological molecular model could be used to monitor the progression of bone metastasis in lung cancer.

Previous studies have rarely reported the correlation between serum BME cytokine levels and bone metastasis in lung cancer. At present, there have been no reports on the application of BME cytokines combined with bone turnover markers in the diagnosis of bone metastasis in lung cancer. In a previous study, Zhou had established a molecular model which involved using four markers to perform immunohistochemical analysis on 105 stage III lung cancer patients, and the model was prospectively validated. It exhibited a prediction sensitivity of $85.7 \%$ and a specificity of $66.7 \%$ [13]. However, the acquisition of tissue samples was limited and could not be monitored continuously or dynamically. In this study, a serum molecular diagnostic model for bone metastasis in lung cancer was established for the first time by combining BME cytokines and bone turnover markers.

Bone biochemical markers that can be observed during bone metastasis include secretory cytokines in the BME, the collagen degradation product tP1NP and $\beta$-CTx, which are formed during bone resorption and bone formation. BME cytokines include CaN, OPG, PTHrP, and IL-6. CaN is a key regulator of gene transcription and differentiation in osteoclasts, and it can promote the differentiation and maturation of osteoclasts through the Ca-CaN-NFATc1 signalling pathway [14]. OPG, a soluble tumour necrosis factor receptor (TNFR), can inhibit the apoptosis of tumour cells by blocking the apoptotic pathway of TRAIL, thereby promoting their survival and growth [15]. On the other hand, OPG can inhibit the function and formation of osteoclasts by inhibiting the RANKL signalling pathway [16]. In addition, OPG plays a key role in promoting angiogenesis [17]. The cytokine IL-6 is a polypeptide with multifunctional biological activity. IL- 6 can regulate the balance between bone formation and bone resorption [18]. This study showed that the serum level of $\mathrm{CaN}$ had a high diagnostic value for bone metastasis in lung cancer, which is consistent with the recent report that the serum $\mathrm{CaN}$ level has a high positive correlation with bone metastasis in breast cancer patients [19]. In prostate, breast, and lung cancer, serum OPG levels of bone metastasis patients tend to be higher than those of non-bone metastasis patients [2022]. The multitude of biological effects of IL- 6 produced more interference factors in the diagnosis and monitoring of bone metastasis, so the serum level of IL- 6 had no diagnostic value $[7,18]$. Serum PTHrP levels had a good application value in the early diagnosis and progression monitoring of bone metastasis in lung cancer, and the combined diagnostic value of OPG and PTHrP was higher. Previous studies have found that high PTHrP levels are significantly associated with high bone metastasis rates and low median survival rates in patients with lung cancer, which is consistent with our results [23-25].

The bone turnover markers tP1NP and $\beta$-CTx are products of bone metabolism, and they are important serum biochemical markers of bone formation and bone resorption, respectively [26, 27]. In the process of bone metastasis, once the balance of bone formation and bone absorption has been destroyed, the concentrations of tP1NP and $\beta-C T x$ in the blood will increase significantly [28-30]. The present study 

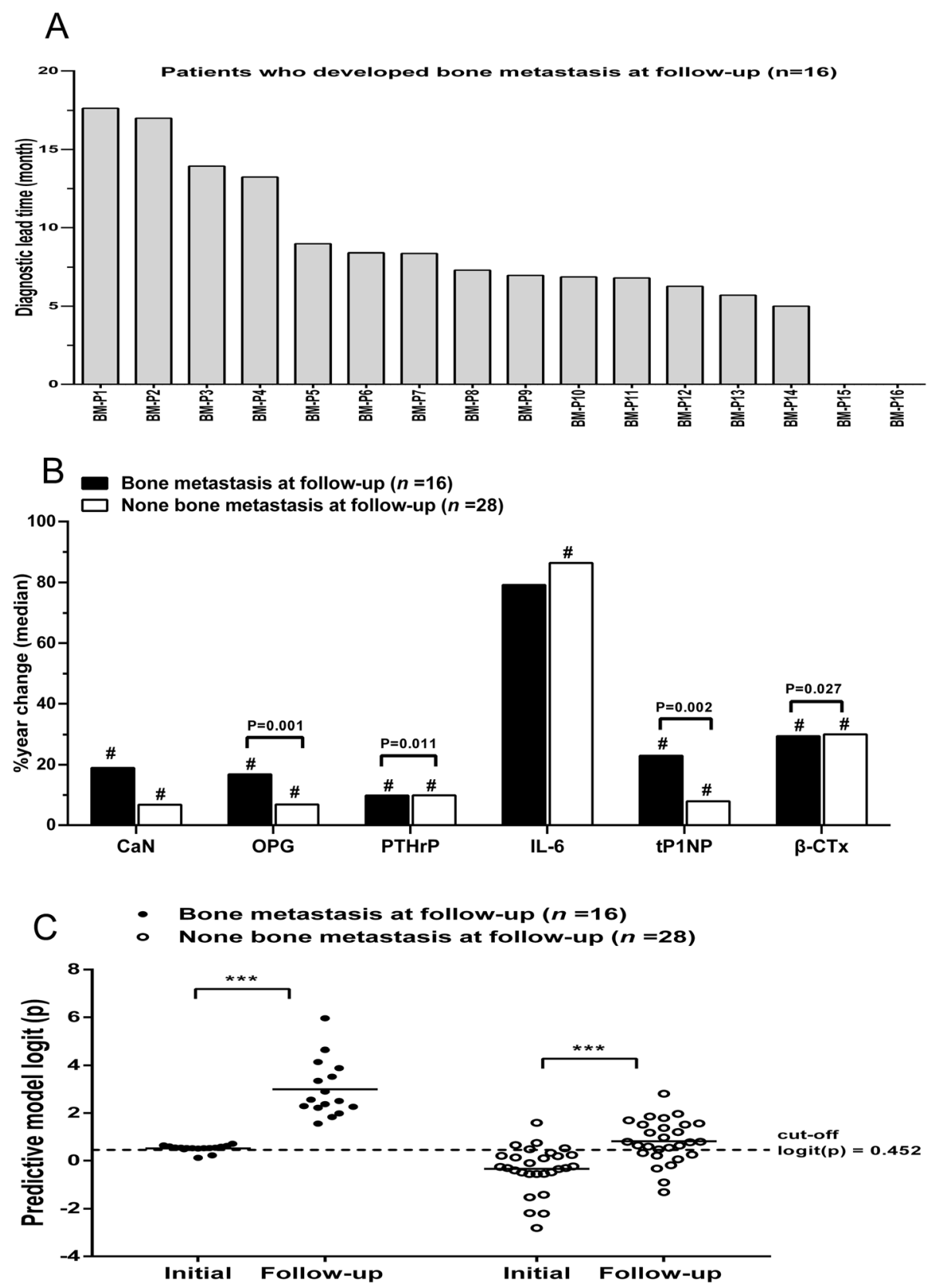

Fig. 6 Evaluation of the progression monitoring abilities of the serum levels of bone biochemical markers and the diagnostic model. a The lead time of the diagnostic model of bone metastasis was 5.00-17.63 months, compared with the date of bone scan diagnosis for 16 patients with stage IV lung cancer who developed bone metastasis during follow-up. $\mathbf{b}$ The changes in the serum levels of bone biochemical markers in patients with lung cancer who developed or remained free of bone metastasis during follow-up. For each patient, the changes in the serum levels of bone biochemical markers during follow-up were calculated as [\% year change (median)] = [(follow-up level - initial level) $/(2 *$ initial level)]. The bars represent the $\%$ year changes (median). The actual $P$ value indicated in the figure refers to a comparison of the $\%$ year changes between the two groups. The \# indicated in the figure represents $P<0.05$ : comparison between the follow-up levels and the initial levels within each group separately. $\mathbf{c}$ The diagnostic model logit $(p)$ of the initial levels and follow-up levels of patients with lung cancer who developed or remained free of bone metastasis during follow-up. The horizontal line on the figure represents the median of the scatter plots for this group. ${ }^{* * *} P<0.001$, comparison between the follow-up levels and the initial levels in each group separately

suggests that serum levels of tP1NP and $\beta$-CTx can be used for the prediction and progression monitoring of bone metastasis in lung cancer.
The correlation analysis of cytokines showed a strong positive correlation between CaN and PTHRP $(r=0.674$, $P<0.001$ ) (Fig. 3A), indicating that in the BME, tumour 
cells interact with other cells through BME cytokines. Interestingly, the cytokine PTHrP also showed a moderately positive correlation with the bone turnover marker tP1NP $(\mathrm{r}=0.428, P<0.001)$ (Fig. 3B). The correlation between BME cytokines and bone turnover markers suggests that the combination of these two factors may be an effective method for improving the early diagnosis of bone metastasis. However, serum bone biochemical markers are susceptible to individual differences and seasonal variations [31, 32]. Therefore, a combination of these bone biochemical markers and bone scan techniques may be more conducive to the diagnosis of bone metastasis in lung cancer.

Multivariate logistic regression analysis revealed that the serum BME cytokines OPG and PTHrP and the bone turnover markers $\mathrm{tP} 1 \mathrm{NP}$ and $\beta$-CTx were independent diagnostic factors for bone metastasis. Furthermore, a mathematical model was established to combine these indicators, so as to predict the risk of bone metastasis in patients with advanced lung cancer more accurately: $\operatorname{logit}(\mathrm{p})=-8.4988+0.0211 * \mathrm{tP} 1 \mathrm{NP}+0.0079 *$ PTHrP $+0.0016^{*}$ OPG $+0.0021^{*} \beta-C T x$. The AUC of this model reached as high as 0.940 . When logit $(\mathrm{p})>0.452$, the specificity and sensitivity of bone metastasis diagnosis in lung cancer are 85.8 and $89.7 \%$. Prospective verification of this model revealed that its sensitivity and specificity for the diagnosis of bone metastasis in lung cancer were 87.5 and $85.7 \%$, respectively, and the Kappa value was 0.770 , which was highly consistent with the actual results. A follow-up study of 44 patients for a minimum of 2 years confirmed that the model could accurately predict the occurrence of bone metastasis in lung cancer, and the prediction of bone metastasis was 9.46 (5.00-17.63) months earlier than medical imaging evidence appeared (Fig. 6A). Additionally, it was found that changes in serum OPG, PTHrP, tP1NP, $\beta$-CTx, and the diagnostic model logit $(\mathrm{p})$ were positively correlated with the progression of bone metastasis, suggesting that the diagnostic model could be used to monitor bone metastasis progression. These results further prove the validity of the diagnostic model.

While these results present novel and innovative diagnostic solutions, the limitations of this study warrant clear recognition. The molecular model was established to predict the risk of bone metastasis in resected stage IV lung cancer in order to screen the patients with a high risk of bone metastasis for early intervention. Therefore, the method might be more suitable for predicting the risk of bone metastasis in stage IV patients than those in other stages before medical imaging evidence appears. In the future, the diagnostic value and prognostic value of this model will require validation through multicentre studies with larger sample sizes and retrospective or prospective studies at different disease stages, so as to improve the efficacy of this lung cancer bone metastasis diagnostic model.

\section{Conclusion}

The serum molecular diagnostic model composed of BME cytokines (OPG and PTHrP) and bone turnover markers (tP1NP and $\beta-C T x)$ can assist with the diagnosis and progression monitoring of bone metastasis in lung cancer. These bone biomarkers can be continuously (once every three to six months, depending on the patient's situation) detected in the sera of lung cancer patients during disease progression, and a continuous, dynamic assessment of bone metastasis risk can be conducted based on the prediction model. If bone metastasis can be predicted early enough, then effective preventative measures could be taken, which may improve the life quality of the lung cancer patients. This model may provide a simple, non-invasive, and reliable method for the early screening of bone metastases in lung cancer patients.

\section{Supplementary information}

Supplementary information accompanies this paper at https://doi.org/10. 1186/s12885-020-07046-2.

Additional file 1: Supplementary Table 1. The clinical characteristics of 205 lung cancer patients for establishment of the diagnostic model of bone metastasis analysis.

Additional file 2: Supplementary Table 2. The basic information of 44 lung cancer patients for prospective validation analysis.

Additional file 3: Supplementary Table 3. Correlations of serum levels for BME cytokines and bone turnover markers in 205 patients with primary lung cancer stage IV.

Additional file 4: Supplementary Table 4. Univariate and multivariate analysis of diagnostic risk factors associated with bone metastasis diagnosis in 205 patients with lung cancer.

\section{Abbreviations}

BME: Bone microenvironment; CaN: Calcineurin; OPG: Osteoprotegerin; PTHrP: Parathyroid hormone related-peptide; IL-6: Interleukin-6; tP1NP: Total amino-terminal propeptide of type I procollagen; $\beta$-CTx: Carboxy-terminal crosslinked telopeptide of type I collagen $\beta$ isomer; AUC: Area under the curve; ROC: Receiver operating characteristics; Cl: Confidence interval; OR: Odds ratio

\section{Acknowledgements}

Not applicable.

\section{Authors' contributions}

X.Y.T designed the study, carried out the experiment and wrote the manuscript. L.R.W and L.M.H collected and analyzed the data. D.L.M and Y.Z.D conceived the original idea and analyzed the results. The authors read and approved the final manuscript.

\section{Funding}

This study was supported by the Science and Technology Development Fundation of Shanghai Pudong New District (No. PKJ2014-Y06 to Y.Z.D), the East Campus of Shanghai Sixth People's Hospital's project (No. DY2018009 to X.Y.T). The funders had a key role in study design, data collection and analysis, decision to publish, and preparation of the manuscript. 


\section{Availability of data and materials}

Please contact the corresponding author for data request.

\section{Ethics approval and consent to participate}

The study was performed in accordance with guidelines approved by the Ethics Committee of Shanghai Sixth People's Hospital East Affiliated to Shanghai Jiao Tong University (Shanghai, China). The study was performed in accordance with guidelines approved by the Ethics Committee of Shanghai Sixth People's Hospital East Affiliated to Shanghai Jiao Tong University (Shanghai, China). A written informed consent to participate in the study was obtained from each subject in accordance with the declaration of Helsinki principles. All study participants approved the storage of their frozen serum specimens, for research purposes, in our laboratory.

\section{Consent for publication}

Not applicable.

\section{Competing interests}

The authors declare that they have no conflict of interest.

Received: 13 April 2019 Accepted: 6 June 2020

Published online: 16 June 2020

\section{References}

1. Bai SB, Liu DZ, Cheng Y, Cui H, Liu M, Cui MX, Zhang BL, Mei QB, Zhou SY. Osteoclasts and tumor cells dual targeting nanoparticle to treat bone metastases of lung cancer. Nanomedicine. 2019;21:102054.

2. Hernandez RK, Wade SW, Reich A, Pirolli M, Liede A, Lyman GH. Incidence of bone metastases in patients with solid tumors: analysis of oncology electronic medical records in the United States. BMC Cancer. 2018;18(1):44.

3. The Youth Specialists Committee of Lung Cancer, Beijing Medical Award Foundaton, Chinese Lung Cancer Union. Expert Consensus on the Diagnosis and Treatment of Bone Metastasis in Lung Cancer (2019 Version). Zhongguo Fei Ai Za Zhi. 2019;22(4):187-207.

4. Murakami A, Matsuda M, Harada Y, Hirata M. Phospholipase C-related, but catalytically inactive protein (PRIP) up-regulates osteoclast differentiation via calcium-calcineurin-NFATc1 signaling. J Biol Chem. 2017;292(19):7994-8006.

5. Kan C, Vargas G, Pape FL, Clézardin P. Cancer cell colonisation in the bone microenvironment. Int J Mol Sci. 2016;17(10):1674.

6. Yoneda T, Hiraga T. Crosstalk between cancer cells and bone microenvironment in bone metastasis. Biochem Biophys Res Commun. 2005;328(3):679-87.

7. Ara T, Declerck YA. Interleukin-6 in bone metastasis and cancer progression. Eur J Cancer. 2010:46(7):1223-31.

8. Takada J, Dinavahi R, Miyauchi A, et al. Relationship between P1NP, a biochemical marker of bone turnover, and bone mineral density in patients transitioned from alendronate to romosozumab or teriparatide: a post hoc analysis of the STRUCTURE trial. J Bone Miner Metab. 2020;38(3):310-5.

9. Wang Z, Lu Y, Qiao D, Wen X, Zhao H, Yao Y. Diagnostic and prognostic validity of serum bone turnover markers in bone metastatic non-small cell lung cancer patients. J Bone Oncol. 2015;4(3):85-91.

10. D'Oronzo S, Brown J, Coleman R. The value of biomarkers in bone metastasis. Eur J Cancer Care (Engl). 2017;26(6). https://doi.org/10.1111/ecc 12725.

11. Seibel MJ. Clinical use of markers of bone turnover in metastatic bone disease. Nat Clin Pract Oncol. 2005;2(10):504-33.

12. Roodman GD. Mechanisms of bone metastasis. N Engl J Med. 2004;350(16): 1655-64.

13. Zhou Z, Chen ZW, Yang XH, Shen L, Ai XH, Lu S, Luo QQ. Establishment of a biomarker model for predicting bone metastasis in resected stage III nonsmall cell lung cancer. J Exp Clin Cancer Res. 2012;31(1):34.

14. Minami T, Jiang S, Schadler K, Suehiro J, Osawa T, Oike Y, Miura M, Naito M, Kodama T, Ryeom S. The calcineurin-NFAT-angiopoietin-2 signaling axis in lung endothelium is critical for the establishment of lung metastases. Cell Rep. 2013;4(4):709-23.

15. Neville-Webbe HL, Cross NA, Eaton CL, Nyambo R, Evans CA, Coleman RE, Holen I. Osteoprotegerin (OPG) produced by bone marrow stromal cells protects breast cancer cells from TRAIL-induced apoptosis. Breast Cancer Res Treat. 2004;86(3):269-79.

16. Ikebuchi $Y$, Aoki S, Honma M, Hayashi M, Sugamori Y, Khan M, Kariya Y, Kato G, Tabata Y, Penninger JM, Udagawa N, Aoki K, Suzuki H. Coupling of bone resorption and formation by RANKL reverse signalling. Nature. 2018; 561(7722):195-200.

17. Cross SS, Yang Z, Brown NJ, Balasubramanian SP, Evans CA, Woodward JK, Neville-Webbe HL, Lippitt JM, Reed MW, Coleman RE, Holen I. Osteoprotegerin (OPG)--a potential new role in the regulation of endothelial cell phenotype and tumour angiogenesis? Int J Cancer. 2006; 118(8):1901-8.

18. Itoh S, Udagawa N, Takahashi N, Yoshitake F, Narita H, Ebisu S, Ishihara K. A critical role for interleukin-6 family-mediated Stat3 activation in osteoblast differentiation and bone formation. Bone. 2006;39(3):505-12.

19. Zhuang X, Zhang H, Li X, Li X, Cong M, Peng F, Yu J, Zhang X, Yang Q, Hu $G$. Differential effects on lung and bone metastasis of breast cancer by Wnt signalling inhibitor DKK1. Nat Cell Biol. 2017;19(10):1274-85.

20. Nyambo R, Cross N, Lippitt J, Holen I, Bryden G, Hamdy FC, Eaton CL. Human bone marrow stromal cells protect prostate cancer cells from TRAILinduced apoptosis. J Bone Miner Res. 2004;19(10):1712-21.

21. Holen I, Cross SS, Neville-Webbe HL, Cross NA, Balasubramanian SP, Croucher PI, Evans CA, Lippitt JM, Coleman RE, Eaton CL. Osteoprotegerin (OPG) expression by breast cancer cells in vitro and breast tumours in vivo-a role in tumour cell survival? Breast Cancer Res Treat. 2005;92(3):207-15.

22. Zhu B, Kejing $S$, Hang $Y, X u Y$. The clinical assessment on lesions shown $\sim(99 \mathrm{~m})$ Tc-MDP whole body bone scan and determination of serum levels of OPG, BSP and TRACP-5b in lung Cancer patients with bone metastasis. J Radioimmunol. 2013;26(5):545-7.

23. Henderson MA, Danks JA, Slavin JL, Byrnes GB, Choong PF, Spillane JB, Hopper JL, Martin TJ. Parathyroid hormone-related protein localization in breast cancers predict improved prognosis. Cancer Res. 2006;66(4):2250-6.

24. Hiraki A, Ueoka H, Bessho A, Segawa Y, Takigawa N, Kiura K, Eguchi K, Yoneda T, Tanimoto M, Harada M. Parathyroid hormone-related protein measured at the time of first visit is an indicator of bone metastases and survival in lung carcinoma patients with hypercalcemia. Cancer. 2002;95(8): 1706-13.

25. Wu CE, Wang CW, Huang WK, Yang CT, Wu YC, Hou MM, Hsieh CH, Hsieh JJ, Cheng HY, Hsu T, Chang JW. Cytoplasmic and nuclear parathyroid hormone-related proteins are opposing prognostic factors in patients with non-small-cell lung cancer who have undergone curative resection. Jpn J Clin Oncol. 2015;45(3):267-73.

26. Henriksen K, Karsdal MA, Martin TJ. Osteoclast-derived coupling factors in bone remodeling. Calcif Tissue Int. 2014;94(1):88-97.

27. Terpos E, Kiagia M, Karapanagiotou EM, Charpidou A, Dilana KD, Nasothimiou E, Harrington KJ, Polyzos A, Syrigos KN. The clinical significance of serum markers of bone turnover in NSCLC patients: surveillance, management and prognostic implications. Anticancer Res. 2009;29(5):16517.

28. De la Piedra C, Alcaraz A, Bellmunt J, et al. Usefulness of bone turnover markers as predictors of mortality risk, disease progression and skeletalrelated events appearance in patients with prostate cancer with bone metastases following treatment with zoledronic acid: TUGAMO study. $\mathrm{Br}$ J Cancer. 2013;108(12):2565-72.

29. Vives V, Cres G, Richard C, Busson M, Ferrandez Y, Planson AG, Zeghouf M, Cherfils J, Malaval L, Blangy A. Pharmacological inhibition of Dock5 prevents osteolysis by affecting osteoclast podosome organization while preserving bone formation. Nat Commun. 2015;6:6218.

30. Brown JE, Coleman RE. Denosumab in patients with cancer-a surgical strike against the osteoclast. Nat Rev Clin Oncol. 2012;9(2):110-8.

31. D'Oronzo S, Stucci S, Tucci M, Silvestris F. Cancer treatment-induced bone loss (CTIBL): pathogenesis and clinical implications. Cancer Treat Rev. 2015; 41(9):798-808

32. Makhoul I, Montgomery CO, Gaddy D, Suva $\sqcup$. The best of both worlds managing the cancer, saving the bone. Nat Rev Endocrinol. 2016;12(1):29-42.

\section{Publisher's Note}

Springer Nature remains neutral with regard to jurisdictional claims in published maps and institutional affiliations. 